\title{
VIRTUAL REALITY SIMULATION AS THERAPY FOR POSTTRAUMATIC STRESS DISORDER (PTSD)
}

\author{
Dr. S. Smys, \\ Professor, \\ Department of CSE, \\ RVS Technical Campus, \\ Coimbatore, India. \\ Email:smys375@gmail.com
}

\author{
Dr. Jennifer S. Raj, \\ Professor, \\ Department of ECE, \\ Gnanamani College of Technology, \\ Namakal, India. \\ Email: jennifer.raj@gmail.com
}

\author{
Mr. N. Krishna raj, \\ Assistant Professor, \\ Department of Information Technology, \\ Mahalingam College of Engineering and Technology, \\ Pollachi, India \\ Email: krishna.rajtce@gmail.com
}

\begin{abstract}
Virtual reality (VR) technology has the potential to make a person experience anything, anytime, anywhere. It has the ability to influence the human brain that it assumes to be present somewhere that it is really not. In this paper, we exploit this application of the VR technology to simulate virtual environments that can help with PTSD therapy for people affected by trauma due to accident, war, sexual abuse and so on. Several sensors are used to gather the user movements on a motion platform and replicate it in the virtual environment with the help of a Raspberry Pi board and Unreal Developer's kit. It has flexible interfaces that the clinician can modify the virtual environment according to the requirement for the patient.
\end{abstract}

Keywords: Virtual Reality, Motion Platform, Unreal Developer's Kit, Sense Hat, Raspberry Pi, PTSD

\section{INTRODUCTION}

Virtual Reality is a computer based simulation of an environment that can mimic physical existence in real or imagined worlds. VR technology is gaining notable acceptance in the field of psychiatry, gaming and education. Several clinical trials are being conducted for therapies for treating psychiatric disorders. VR systems consists of VR headset, computer, motion platform, chairs, gloves, sensors, head-mounted goggles, speakers and so on to create a complete virtual setup. A 4D VR system is inclusive of motion chair or platform that heightens reality for users with integrated movement according to the VR video content. 
PTSD conditions can occur after events like serious accident, war, physical or sexual assault, abuse, health problem, trauma at work and so on. VR has been used in the field of science and medicine since 1990s [1]. VR has astonishing potential to help people suffering from PTSD and phobia. Traditionally, therapy would be provided by qualified physicians with a range of physical activities that could create fatigue and monotony. With VR, people can face different simulation situations virtually and can be instructed on ideal ways to respond to those situations. Thereby it improves the situation handling capability of the individual in reality [2].

With the addition of higher level of sensors, peripherals and devices, the overall performance and VR experience is enriched. In the recent days VR technology is becoming cheaper, portable and flexible. It is also being used in a wide range of environments. Using VR and android platform, several games are developed to treat psychological issues and mend motor functions. VR simulation is used to distract patients when they undergo painful treatment. VR is also used in treating obesity and eating disorders by promoting healthy eating practises with the help of convincing avatars [3]. Yin et al [4] has instituted that VR technology has no major side effects and is safe for the users compared to the traditional therapy techniques that may cause fatigue.

\section{RELATED WORK}

Several research and testing has been done on VR environments and tools. Research has proved Virtual Reality Simulation to have a positive outcome on the treatment. Evidence-based treatments are established and provided for patients with PTSD condition. Many cutting edge research has been undertaken on the VR devices to enhance them for a realistic virtual experience. VR gives an improved response in cognitive fidelity rather than physical fidelity. VR Rehabilitation programs have proved to be more efficient than the traditional methods $[5]$.

Research has been done on VR simulation methods to create virtual environment to promote quality of life in old age [6]. The work focuses on creating VR based games to engage the elderly so as to improve their health as well as quality of life. It allows the controlling of an Unmanned Aerial Vehicle (UAV) with just body movements with the aid of Body Area Network. Unity 3D game engine is used for simulation. It is instigated on an android smartphone and a Google cardboard headset. The application flow diagram of this system is shown in Figure 1. 


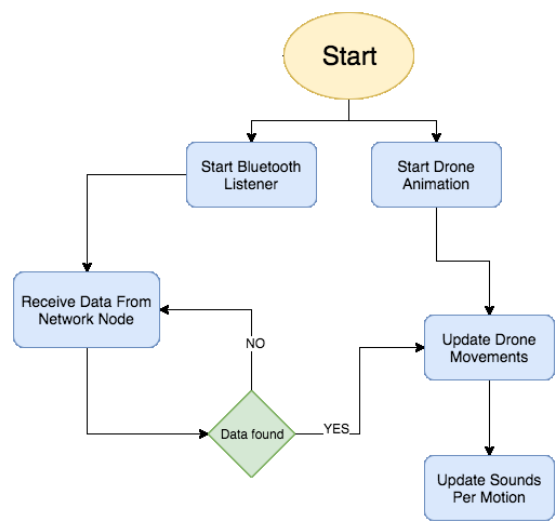

Figure 1 Application Flow Diagram

VR and Cognitive-Behavioural therapy has been tested for people with driving anxiety and aggression [7]. Results show that hyperarousal in during driving decreased by $69 \%$, aggressiveness by $29 \%$, and risky driving by $21 \%$. Lee, Chang Ha [8] in his paper, proposed location aware speaker technology to enhance the VR environment with the use of Raspberry Pi and Beacon. This allows the estimation of distance between speakers using Received Signal Strength Indication (RSSI). Jiawang Bai et al. [9] aims in designing a VR robot based on an android platform. It improves the human-computer interaction with pan, tilt and zoom features using binocular camera along with automatic speech recognition.

De Oliveira et al. [10] proposed a VR system along with fuzzy logic to assist people with PTSD. Fuzzy logic is used to alter the inputs based on inference and thereby control the system. This allows in identifying patterns of anxiety depending on the heart rate as it is the finest feature of fuzzy logic. Sánchez et al. [11] has presented a virtual system for rehabilitation with the help of electromyography sensors that is used as a smart band device to strengthen the hands and shoulder portion. It presents a virtual landscape scenario for the user to drive in a specific route. Precautions are taken in such a way that stressful scenarios are dodged during the therapy.

\section{PROPOSED WORK}

This paper proposes a simple VR simulation based system that consists of a VR headset, speaker, VR gloves and a motion platform. The trauma specific stimulus - scenarios and features are fed into the VR headset through the raspberry pi module and is used for treating the candidates with PTSD. It can also be modified by the clinician as to match the patient's trauma experience. Figure 2 shows the block diagram of the proposed VR simulation system. 


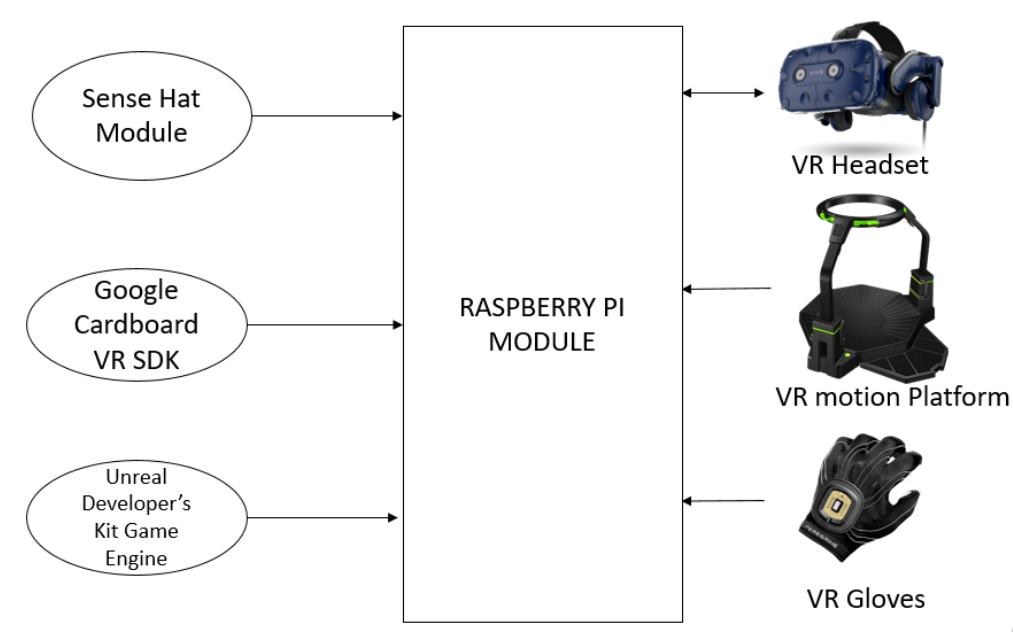

Figure 2 Block Diagram of the VR Simulation Module

\subsection{Raspberry Pi Module}

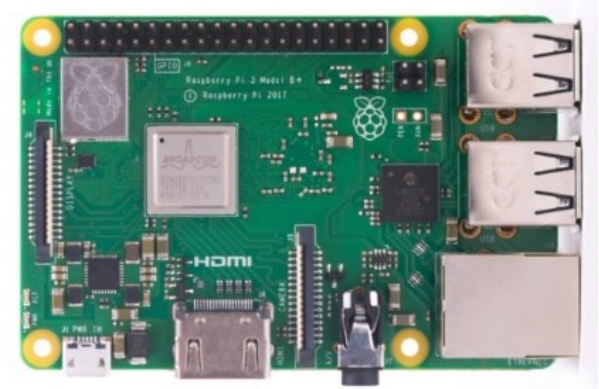

Figure 3 Raspberry Pi 3

Raspberry Pi Module is a portable single board computer that uses Broadcom BCM2837 System on Chip. The CPU used is $1.2 \mathrm{GHz} 4 \mathrm{x}$ ARM Cortex-A53 and has a 1GB RAM. It acts as the main processing core of the VR system and works in processing the user movements in the virtual environment. It links the software and hardware modules to create the complete VR experience.

\subsection{Sense HAT Module for Raspberry Pi}




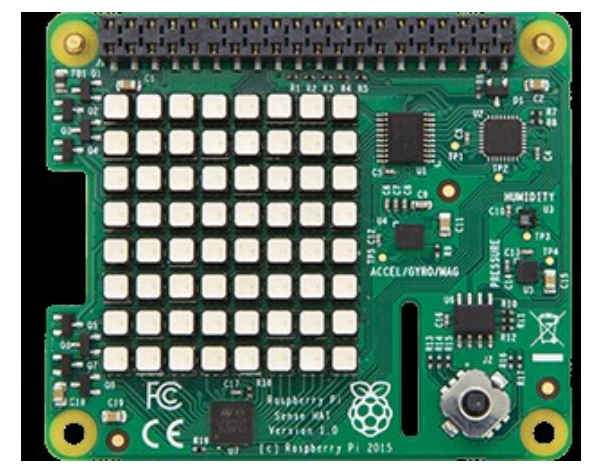

Figure 4 Sense HAT for Raspberry Pi

The Sense HAT for raspberry pi is a complete array of sensors that helps the user to interact with the environment digitally. It comprises of sensors like accelerometer, magnetometer, gyroscope, barometer and sensors that can measure humidity and temperature. It also has an 8x8 LED matrix display and a small 5 button joystick.

\subsection{Unreal Developer's Kit (UDK) Game Engine}

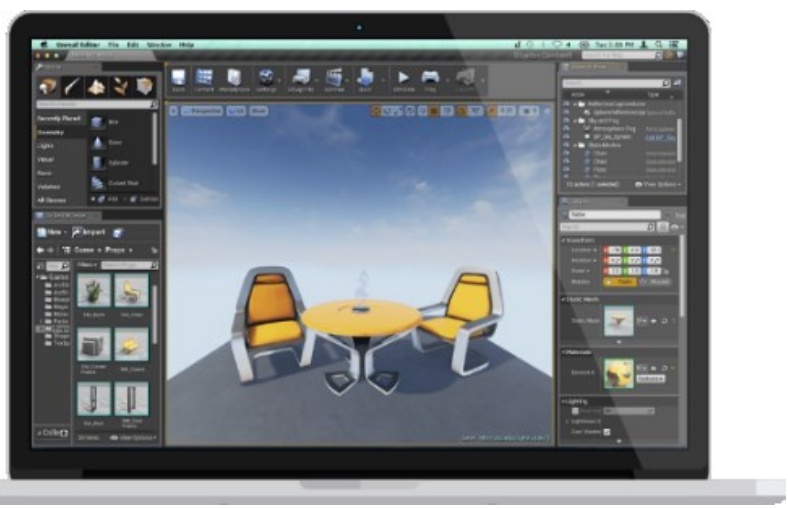

Figure 5 UDK User Interface

UDK is a free edition of Unreal Engine. It is a robust software tool that is used for developing crossplatform virtual applications. We use this tool to generate an application with multiple simple virtual environments that would assist in the treatment of patients with PTSD. There are several other game engines 
Journal of Electronics and Informatics (2019)

Vol.01/ No. 01

Pages: 24-34

http://www.irojournals.com/iroei/

https://doi.org/10.36548/jei.2019.1.003

that provide more simpler and complex tools to create the required virtual environment. UDK helps in creating software of varied sizes and can efficiently manage data and collaborate multiple artists and developers.

\subsection{VR headset}

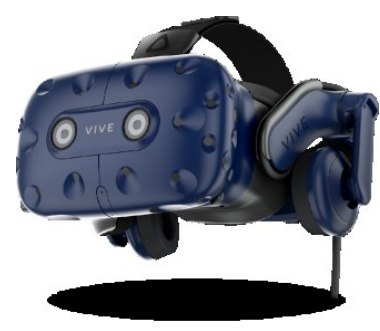

Figure 6 VR Headset

VR headsets comprises of a head-mounted display with audio headset and motion tracking sensor to monitor head movements. Certain VR headsets also monitor eyeball movement of the user. Nintendo was the first company to release VR headset for commercial use in the year 1995. In the proposed system, we use HTC Vive VR headset. This headset is PC compatible and primarily contains motion controllers. Positional tracking is done with the help of laser towers. The headset consists of lenses that provide a wide field display and a relaxed point of focus.

\subsection{VR Gloves}

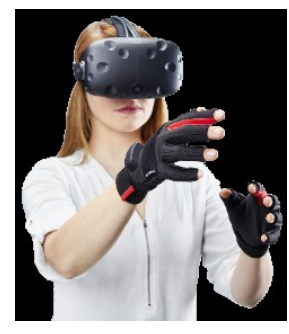

Figure 7 VR Gloves

VR gloves are human-computer interface devices that provide the user a complete virtual experience of touch sensitivity. It allows the user to feel, grab or place objects in the virtual environment. The glove is embedded with sensors like piezoresistive sensor or inertial measurement sensor. Piezoelectric sensors can also 
Journal of Electronics and Informatics (2019)

Vol.01/ No. 01

Pages: 24-34

http://www.irojournals.com/iroei/

https://doi.org/10.36548/jei.2019.1.003

be used to harvest energy during motion. Soft actuators that are small, flexible and of light weight are preferred for use in these gloves.

\subsection{VR Motion Platform}

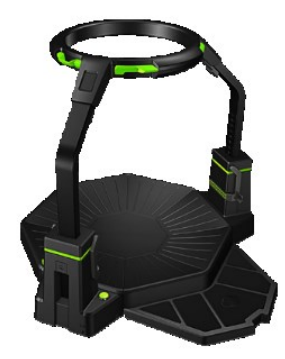

Figure 8 VR Motion Platform

The VR motion platform is a device that can be used for industrial applications, training simulation and gaming purposes. It tracks the foot location of the user accurately and offers independent body and foot head tracking. We use the KAT Walk VR motion platform that can handle up-to $130 \mathrm{Kgs}$ of weight and person of height ranging from 140-195cms. It consents with user movements like running, walking, sitting, squatting, crouching, and jumping in VR environment. It has a sleek and adaptive tension design and allows free range motion with zero motion tracking delay.

\subsection{Signal Acquisition}

Based on the process diagram shown in figure 9, signal acquisition is done with the help of various sensors in the Sense HAT module, VR headset, VR glove and VR Motion Platform. The gathered signals are then collectively sent to the Raspberry pi module from which they are transferred back to the VR headset virtual environment. Depending on the inputs received from these sensors, movements and responses are created in the virtual environment.

\begin{tabular}{|c|c|c|c|}
\hline \begin{tabular}{|l|l|}
\hline $\begin{array}{c}\text { Sense HAT module } \\
\text { signals }\end{array}$ \\
\cline { 2 - 3 } $\begin{array}{c}\text { VR Headset sensor } \\
\text { signals }\end{array}$
\end{tabular} & $\begin{array}{c}\text { VR Glove sensor } \\
\text { signals }\end{array}$ & $\begin{array}{c}\text { Motion Platform } \\
\text { sensor signals }\end{array}$ \\
\hline & $\begin{array}{c}\text { RASPBERRY PI } \\
\text { MODULE }\end{array}$ & \\
\hline
\end{tabular}




\section{Figure 9 Signal Acquisition Unit}

\subsection{Interface Development}

The Unreal Developer's Kit game engine and Google VR software development kit are used in the interface development for the proposed VR system. UDK helps in creation of virtual environments and behaviour programming. A database of VR scenarios are created along with the environmental sounds that match with the situation in order to provide a realistic sensation. Another database that consists of a predefined set of gestures that are compared with the user gestures is also available in the system. Android platform is used in the VR headset that allows communication of the virtual scenario with the user. The combination of the Virtual database, UDK game engine and android platform acts as the software interface for the device and assists in the application execution.

\section{TESTS AND RESULT}

A usability test - Single Ease Question (SEQ) is conducted on the participants to analyse their experience with the system. A set of 5 questions are asked to the users and the answers are rated on a scale of 1-5. The virtual system obtained a score of 4.36 . Hence we can determine that it is an idea tool to be used the rehabilitation process.

Table 1 Usability Test Response

Question

Mean Response (1-5)

1. How do you rate the reality of the virtual environment?

4.2

2. Did this system meet your expectations? 
Journal of Electronics and Informatics (2019)

Vol.01/ No. 01

Pages: 24-34

http://www.irojournals.com/iroei/

https://doi.org/10.36548/jei.2019.1.003

3. Did you feel comfortable using the system?

4.6

4. How do you rate the clarity of information provided by the system?

4.4

5. How do you rate the system overall with regard to your experience?

4.3

Overall Average Score

4.36

\section{CONCLUSION}

A complete trauma rehabilitation solution is developed by this system using Raspberry Pi, Android and UDK Game engine and implemented using VR headset, gloves and motion platform. The design involves user friendly approach that allows the clinicians to alter the virtual environment based on the individual patient requirements. Future work involves adding more sensors to the system to monitor heart rate and pressure of the user on exposure to different scenarios.

\section{References}

[1] Freeman, Daniel, Sarah Reeve, A. Robinson, Anke Ehlers, David Clark, Bernhard Spanlang, and Mel Slater. "Virtual reality in the assessment, understanding, and treatment of mental health disorders." Psychological medicine 47, no. 14 (2017): 2393-2400.

[2] Dascal, Julieta, Mark Reid, Waguih William IsHak, Brennan Spiegel, Jennifer Recacho, Bradley Rosen, and Itai Danovitch. "Virtual reality and medical inpatients: a systematic review of randomized, controlled trials." Innovations in clinical neuroscience 14, no. 1-2 (2017): 14.

[3] Wiederhold BK. VRMC: The Virtual Reality Medical Center [website] The potential for virtual reality to improve health care. 2006. [Accessed May 3, 2016]. 
Journal of Electronics and Informatics (2019)

Vol.01/ No. 01

Pages: 24-34

http://www.irojournals.com/iroei/

https://doi.org/10.36548/jei.2019.1.003

[4] Yin CW, Sien NY, Ying LA, et al. Virtual reality for upper extremity rehabilitation in early stroke: a pilot randomized controlled trial. Clin Rehabil. 2014;28:1107-1114.

[5] Howard, Matt C. "A meta-analysis and systematic literature review of virtual reality rehabilitation programs." Computers in Human Behavior 70 (2017): 317-327.

[6] Crespo, Andrés Bustamante, Graciela Guerrero Idrovo, Nuno Rodrigues, and António Pereira. "A virtual reality UAV simulation with body area networks to promote the elders life quality." In 2016 1st International Conference on Technology and Innovation in Sports, Health and Wellbeing (TISHW), pp. 1-7. IEEE, 2016.

[7] Zinzow, Heidi M., Johnell O. Brooks, Patrick J. Rosopa, Stephanie Jeffirs, Casey Jenkins, Julia Seeanner, Alyssa McKeeman, and Larry F. Hodges. "Virtual reality and cognitive-behavioral therapy for driving anxiety and aggression in veterans: a pilot study." Cognitive and behavioral practice 25, no. 2 (2018): 296-309.

[8] Lee, Chang Ha. "Location-aware speakers for the virtual reality environments." IEEE Access 5 (2017): 2636-2640.

[9] Bai, Jiawang, Xin Wang, Wenbin Zhang, Chaojian Zhuang, and Mengdi Pan. "Design of virtual reality robot based on android platform." In 2017 Fourth International Conference on Image Information Processing (ICIIP), pp. 1-4. IEEE, 2017.

[10] de Oliveira, Fernando M., Regina S. Lanzillotti, Rosa MEM da Costa, Raquel Gonçalves, Paula Ventura, and Luís AV de Carvalho. "A virtual reality exposure therapy for PTSD patientes controlled by a fuzzy logic system." Revista Carioca de CiÊncia, tecnologia e educação 1, no. 1 (2016).

[11] Sánchez, Z. Andrea, T. Santiago Alvarez, F. Roberto Segura, C. Tomás Núñez, P. Urrutia-Urrutia, L. Franklin Salazar, S. Altamirano, and J. Buele. "Virtual Rehabilitation System Using Electromyographic Sensors for Strengthening Upper Extremities." In Developments and Advances in Defense and Security, pp. 231-241. Springer, Singapore, 2020.

[12] Rizzo, Albert, Judith Cukor, Maryrose Gerardi, Stephanie Alley, Chris Reist, Mike Roy, Barbara O. Rothbaum, and JoAnn Difede. "Virtual reality exposure for PTSD due to military combat and terrorist attacks." Journal of Contemporary Psychotherapy 45, no. 4 (2015): 255-264. 
Journal of Electronics and Informatics (2019)

Vol.01/ No. 01

Pages: 24-34

http://www.irojournals.com/iroei/

https://doi.org/10.36548/jei.2019.1.003

[13] Park, Mi Jin, Dong Jun Kim, Un-joo Lee, Eun Jin Na, and Hong Jin Jeon. "A Literature Overview of Virtual Reality (VR) in Treatment of Psychiatric Disorders: Recent Advances and Limitations." Frontiers in Psychiatry 10 (2019): 505.

[14] Slater, Mel, and Maria V. Sanchez-Vives. "Enhancing our lives with immersive virtual reality." Frontiers in Robotics and AI 3 (2016): 74.

[15] Loucks, Laura, Carly Yasinski, Seth D. Norrholm, Jessica Maples-Keller, Loren Post, Liza Zwiebach, Devika Fiorillo et al. "You can do that?!: Feasibility of virtual reality exposure therapy in the treatment of PTSD due to military sexual trauma." Journal of anxiety disorders 61 (2019): 55-63.

[16] Anam, M., K. Sizemore, H. Mansour, J. Seward, L. Mitchell, C. Shum, B. Richardson, J. Richards, S. Gustin, and Z. Trost. "(309) Virtual Reality Walking for Neuropathic Pain in Spinal Cord Injury: Preliminary Efficacy Findings." The Journal of Pain 20, no. 4 (2019): S51.

[17] Maravilla, Morelia Malina, Angela Cisneros, Alvorado Stoddard, Desiree DeAndrea Scretching, Brian Kyle Murray, and Elissa Redmiles. "Defining virtual reality: Insights from research and practice." iConference 2019 Proceedings (2019). 